\title{
Competition and Synergism between Cholesterol and Cholestanol in Oospore Formation in Phytophthora cactorum
}

\author{
By C. G. ELLIOTT \\ Botany Department, University of Glasgow \\ (Accepted for publication 25 September 1967) \\ SUMMARY
}

Cholesterol promoted oospore formation in Phytophthora cactorum; very few oospores were formed when cholestanol was incorporated in the medium instead. Above a critical concentration of cholesterol, cholestanol competitively inhibited oospore formation by cholesterol when both were added to the medium together. At low cholesterol concentrations, the addition of some cholestanol increased the number of oospores; further addition of cholestanol was inhibitory. Cholesterol and cholestanol stimulated vegetative growth to a similar extent.

\section{INTRODUCTION}

The fungus Phytophthora cactorum (Leb. \& Cohn) Schroet. grows vegetatively on an agar medium containing sucrose, asparagine, mineral salts and thiamine (basal medium); but for sexual reproduction and oospore formation sterols must be added to the medium. Different sterols vary greatly in their effectiveness in promoting reproduction. Some, classified by Elliott, Hendrie \& Knights (I966) as 'active', promote oospore formation, but with others, called 'partially active', only oogonia and antheridia are formed: the contents of the oogonia degenerate and no oospores are formed.

The question arises as to whether a partially active substance added to the medium together with an active one suppresses or enhances the action of the active substance in promoting oospore formation. This paper reports the effect of mixtures of the active substance, cholesterol, and the partially active substance, cholestanol.

\section{METHODS}

The strain IMI 21168 of Phytophthora cactorum was used. The basal medium was that described by Elliott et al. (I966); it was solidified with I \% (w/v) Difco Bactoagar. Cholesterol and cholestanol were dissolved in diethyl ether and added to the medium after it had been autoclaved.

The cholesterol and cholestanol were examined by gas-liquid chromatography (GLC) (see Knights, I967, for details), and each appeared to be at least $99 \%$ pure. To test further for the presence of cholestanol in the cholesterol, the sample was oxidized with Jones's reagent (Bowden et al. 1946) so that the cholesterol was converted to $\Delta^{4}$-cholesten-3,6-dione and cholestanol to cholestan-3-one. A trace of material was detected by GLC which might have been cholestan-3-one (Fig. I), but again the 
maximum possible amount was under I \%. The presence of cholesterol in the cholestanol was tested for by the Liebermann-Burchard reaction. The intensity of colour developed indicated a maximum cholesterol content in the sample of $\mathrm{I} \cdot 65 \%$. However, ultraviolet spectra of the colour reactions of the cholestanol sample and of pure cholesterol were not identical, allowance for dilution being made (Fig. 2). Cholesterol shows a peak at 4 I $5 \mathrm{~m} \mu$ (absent from the cholestanol sample) and the spectrum changes with time (which did not occur with the cholestanol sample); moreover the cholestanol sample showed a weak peak at $455 \mathrm{~m} \mu$, absent from cholesterol. Thus cholesterol content of the cholestanol is likely to be well under $\mathrm{I} \%$.

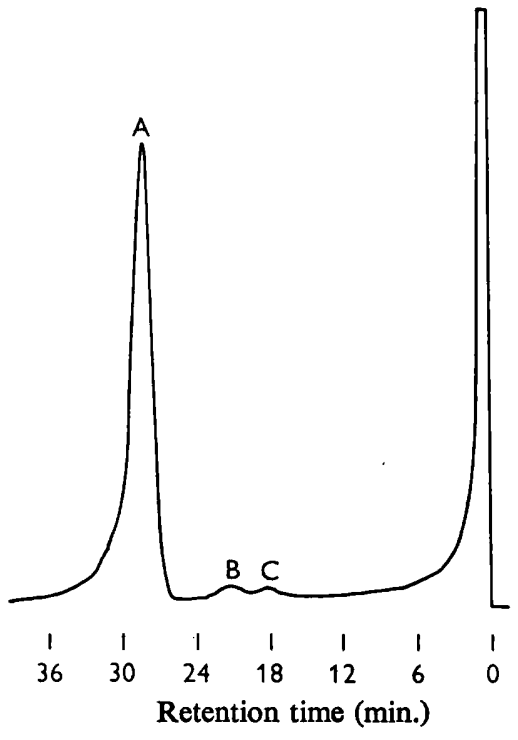

Fig. I

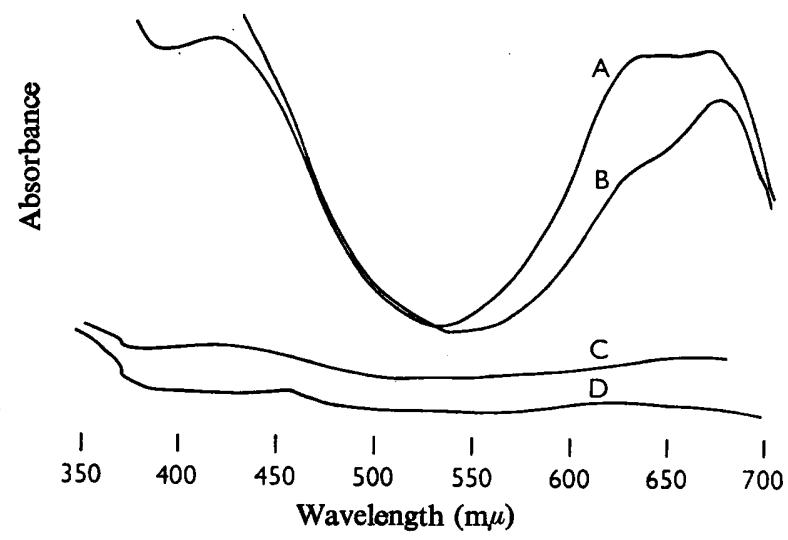

Fig. 2

Fig. I. GLC analysis (on I \% SE-30 at $250^{\circ}$ ) of oxidation product of cholesterol sample. Peak A, cholest-4-ene-3,6-dione; B, cholest-4-ene-3-one; C, cholestan-3-one ?+ unreacted cholesterol.

Fig. 2. Spectra of Liebermann-Burchard reaction mixtures of A, cholesterol ( $1 \cdot 6 \mathrm{mg} . / 2 \mathrm{ml}$.); $\mathrm{B}$, same after about $30 \mathrm{~min}$; $\mathrm{C}$, cholesterol, diluted $\mathrm{I} \cdot 6$ in $100 ; \mathrm{D}$, cholestanol $(6.87 \mathrm{mg}$.) $2 \mathrm{ml}$.).

The fungus was grown in small 'Monax' Petri dishes, $5 \mathrm{~cm}$. diam. (nominal), containing $5 \mathrm{ml}$. medium. The inoculum was a disc $4 \mathrm{~mm}$. diameter cut from a culture growing on basal medium. Incubation was at $24^{\circ}$ in the dark.

Oospore counts were made in cultures 3 weeks old. By this time oogonia either contained oospores, or their contents had degenerated. For counting, the Petri dishes were inverted on the microscope stage and a convenient plane of focus was selected (generally just inside the glass). The Petri dish was traversed under the low-power objective from the outer edge of the colony to the centre, the fine-focus knob being continuously adjusted through one quarter of a turn only.

For statistical tests, the individual oospore counts, $x$, were transformed to $\left(x+\frac{1}{2}\right)^{\frac{1}{2}}$. It is these transformed values which are plotted in Fig. 3 and 4. 


\section{RESULTS}

In the first three experiments (Fig. 3) the concentration of cholestanol was arranged to be $0, \frac{1}{4}, \frac{1}{2}, I, 2, \ldots, 64$ times that of cholesterol (w/w), the amounts of cholesterol added being IO, I and $0.2 \mathrm{mg} . / \mathrm{l}$. in the different experiments. At cholesterol Io mg./l., the addition of even a small proportion of cholestanol decreased the oospore count. At cholesterol $0.2 \mathrm{mg}$./1., the addition of some cholestanol promoted oospore formation; there was a maximum at a ratio 2: I cholestanol:cholesterol, above which the oospore count decreased. At cholesterol I mg./l. no promoting effect of cholestanol was observed, but there was only a small decrease in the oospore count until the ratio cholestanol: cholesterol exceeded 2:I.

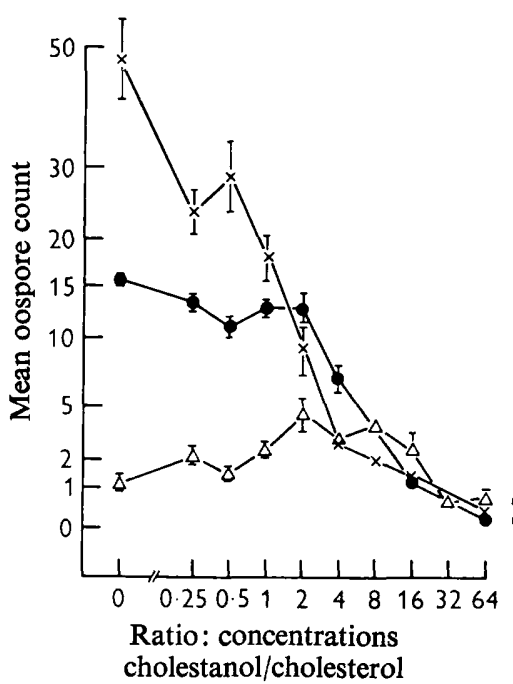

Fig. 3

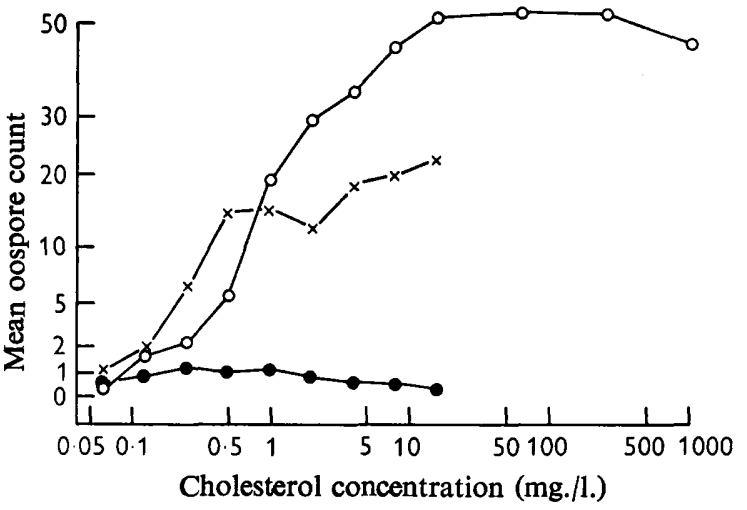

Fig. 4

Fig. 3. Effect on oospore numbers in Phytophthora cactorum of adding cholestanol to medium containing cholesterol: $\times-\times$, ro mg./1. cholesterol; $-0,1 \cdot 0 \mathrm{mg} . / 1$. cholesterol; $\triangle \longrightarrow \triangle, 0.2 \mathrm{mg} . / 1$. cholesterol.

Fig. 4. Oospore numbers of Phytophthora cactorum in relation to concentration of cholesterol $(\mathrm{O}-\mathrm{O})$, cholesterol + cholestanol $\mathrm{I}: 2(\times-\times)$ and cholestanol $(0-0)$. The figures on the scale are to be doubled to give the cholestanol concentration appropriate to each point.

In the next experiment (Fig. 4) the ratio of the amounts of cholestanol and cholesterol was fixed at $2: \mathrm{I}$, but the actual amounts varied. Control cultures were grown with the same amounts of cholesterol and cholestanol separately, and in this experiment the effect of high concentrations of cholesterol on oospore formation was also tested. From Fig. 4 it can be seen that the number of oospores increased with increasing cholesterol concentration up to about $10 \mathrm{mg}$./1. Further increase in the amount brought about no significant change in the number of spores. At the lower concentrations (less than $2 \mathrm{mg}$./1.) the oospores were confined to the outermost part of the colony. At higher concentrations they occurred in increasing numbers in the central region. The curves for oospore number for cholesterol alone and for the cholesterol + cholestanol 
mixture intersected at about cholesterol $0.9 \mathrm{mg}$. $/ 1$. Above this concentration the addition of twice as much cholestanol decreased the number of oospores; below it, the addition of twice as much cholestanol increased it.

In these experiments a very few oospores were found in the cholestanol controls, contrary to our previous experience (Elliott et al. 1966). The use of Monax glassware rather than Pyrex as in our previous work was found not to be the explanation of the difference. The curve relating oospore number to cholestanol concentration (Fig. 4) did appear to have a maximum, but the numbers of spores did not vary significantly between concentrations, and were in all cases very small indeed. No oospores were seen in the controls without either sterol.

\section{Table I. Phytophthora cactorum: mean oospore counts in media with different concentrations of cholesterol and cholestanol}

\begin{tabular}{|c|c|c|c|c|c|}
\hline \multirow{2}{*}{$\begin{array}{l}\text { Concen- } \\
\text { tration } \\
\text { of choles- } \\
\text { tanol } \\
\text { (mg./l.) }\end{array}$} & \multicolumn{5}{|c|}{ Concentration of cholesterol (mg./l.) } \\
\hline & 0.5 & $\mathbf{I}$ & 2 & 5 & 10 \\
\hline $\begin{array}{l}0 \\
1 \\
5\end{array}$ & $\begin{array}{r}6.78 \\
11 \cdot 97 \\
4.00\end{array}$ & $\begin{array}{r}18 \cdot 75 \\
13.78 \\
9.94\end{array}$ & $\begin{array}{l}27 \cdot 31 \\
26 \cdot 03 \\
12 \cdot 72\end{array}$ & $\begin{array}{l}42 \cdot 59 \\
27 \cdot 97 \\
26 \cdot 31\end{array}$ & $\begin{array}{l}56 \cdot 8 I \\
48 \cdot 22 \\
38 \cdot 78\end{array}$ \\
\hline \multicolumn{6}{|c|}{ Analysis of variance (data transformed, $[x+1 / 2]^{\frac{1}{2}}$ ) } \\
\hline & & & D.F. & $\begin{array}{l}\text { Mean } \\
\text { square }\end{array}$ & $F$ \\
\hline \multicolumn{3}{|c|}{ Between cholesterol concentrations } & 4 & 230.99 & $49 \cdot 56 * *$ \\
\hline \multicolumn{3}{|c|}{ Between cholesterol concentrations } & 2 & $67 \cdot 49$ & $13 \cdot 94^{* *}$ \\
\hline \multicolumn{3}{|c|}{ Interaction I } & 2 & $16 \cdot 53$ & $3 \cdot 4 I^{*}$ \\
\hline \multicolumn{3}{|c|}{ Interaction 2} & 6 & $4 \cdot 17$ & - \\
\hline \multicolumn{3}{|c|}{ Between replicate Petri dishes } & 105 & $4 \cdot 84$ & $4 \cdot 22 * *$ \\
\hline \multicolumn{3}{|c|}{ Within dishes } & 360 & $I \cdot 15$ & - \\
\hline
\end{tabular}

Interaction I compares the effect of cholestanol concentration at $0.5 \mathrm{mg} . / 1$. cholesterol with its average effect at the other cholesterol concentrations. Interaction 2 compares the responses to cholestanol at the four higher cholesterol concentrations.

* P 0.05-0.01. ** $P$ very small.

The methods of enzyme kinetics have been applied to the growth of higher plants in response to auxins (Foster, McRae \& Bonner, 1952; McRae, Foster \& Bonner, I953). These authors substituted the rate of elongation for the rate of the reaction in the enzyme system, and the concentrations of auxin and anti-auxin for the concentrations of enzyme substrate and inhibitor. It seemed appropriate to treat the present case similarly, using the oospore count in place of the rate of reaction (v) and concentrations of cholesterol and cholestanol for the concentration of substrate $(s)$ and inhibitor (i), respectively, although the oospore count itself is not strictly a rate.

The data of Table I have been plotted in two ways which are convenient for distinguishing competitive from non-competitive inhibition (Dixon \& Webb, I964), namely $\mathrm{I} / v$ against $\mathrm{I} / s$ (Fig. 5) and $s / v$ against $s$ (Fig. 6). The untransformed means were used for the $v$ values. In Fig. 5 the points at which the lines for cholestanol con. centrations $O$ and $5 \mathrm{mg}$./1. cut the vertical axis do not differ significantly. In Fig. 6 the 
lines for these two concentrations do not differ significantly in slope. These are the results expected in the case of competitive inhibition. For cholestanol I mg./l., the line would appear to lie between the lines for the other two cholestanol concentrations (i.e. 0 and $5 \mathrm{mg}$./1.), but to cross the no-cholestanol line at low cholesterol concentrations where the effect of adding cholestanol to the cholesterol is to increase the oospore count.

Vegetative growth (dry weight of mycelium) in media with cholesterol or cholestanol is compared in Table 2. The results were similar, but cholestanol appeared to be slightly less effective in promoting growth at low concentrations.

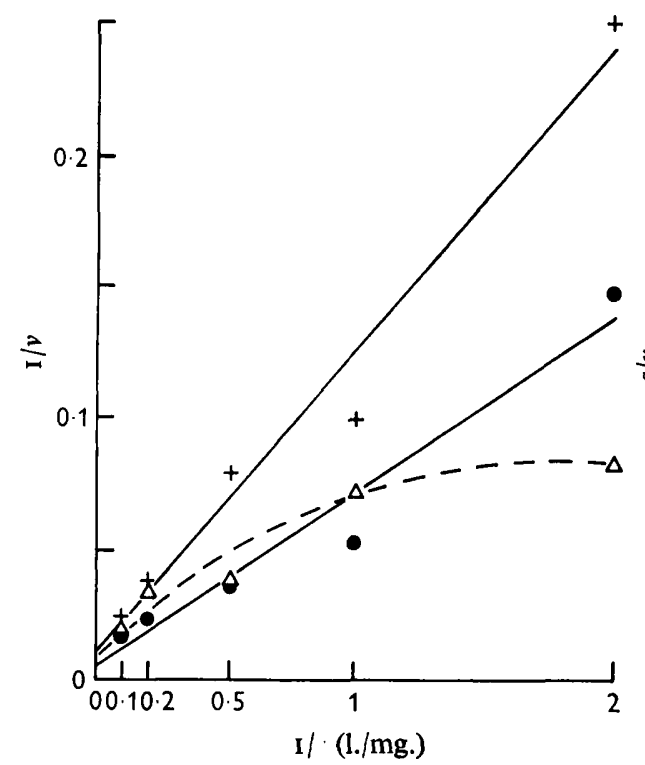

Fig. 5

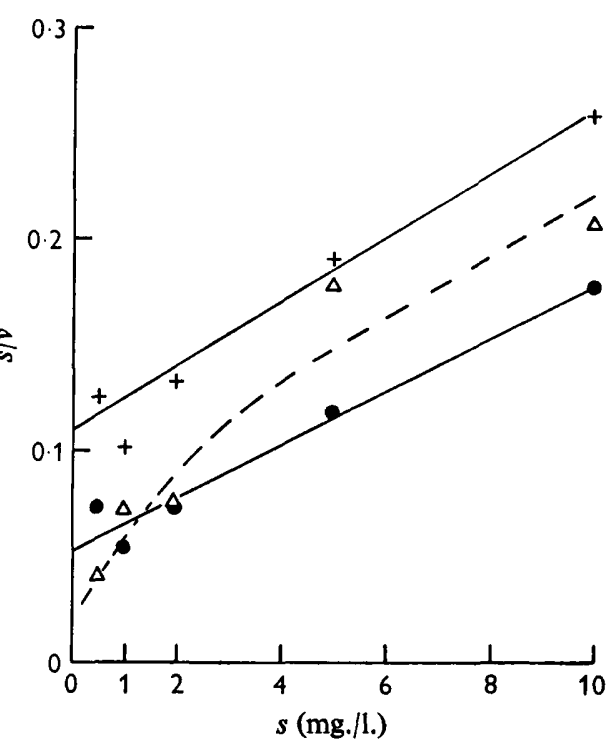

Fig. 6

Fig. 5. Relationship between reciprocal of mean oospore count (I/v) and reciprocal of cholesterol concentration $(\mathrm{I} / \mathrm{s}):+\longrightarrow+, 5 \mathrm{mg}$./ 1 . cholestanol (calculated line); $\triangle---\Delta$, I mg./l. cholestanol (line drawn by eye); - , no cholestanol (calculated line).

Fig. 6. Relationship between ratio of cholesterol concentrations (s) to mean oospore count (v) and cholesterol concentration: symbols as in Fig. 3.

Table 2. Effects of cholesterol and cholestanol on vegetative growth of Phytophthora cactorum

Values given are dry weights (mg.) of mycelium harvested after incubation for 16 days

(Exp. I) or 13 days (Exp. 2) at $24^{\circ}$ in $100 \mathrm{ml}$. flasks containing $25 \mathrm{ml}$. liquid medium.

\begin{tabular}{|c|c|c|c|c|c|}
\hline \multirow[b]{2}{*}{ Experiment } & & \multicolumn{4}{|c|}{ Concentration of sterol (mg./1.) } \\
\hline & & 0 & $2 \cdot 5$ & IO & 40 \\
\hline I & $\begin{array}{l}\text { Cholesterol } \\
\text { Cholestanol }\end{array}$ & $44 \pm 4^{\circ} 0$ & $\begin{array}{l}97 \pm 6 \cdot 7 \\
73 \pm 4 \cdot 9\end{array}$ & $\begin{array}{r}118 \pm 5 \cdot 7 \\
92 \pm 7 \cdot 5\end{array}$ & $\begin{array}{l}\text { I IO } \pm 4 \cdot \text { I } \\
\text { I I I } \pm 3 \cdot 9\end{array}$ \\
\hline 2 & $\begin{array}{l}\text { Cholesterol } \\
\text { Cholestanol }\end{array}$ & $50 \pm 8 \cdot 5$ & $\begin{array}{l}74 \pm 5 \cdot 2 \\
75 \pm 4 \cdot 5\end{array}$ & $\begin{array}{l}92 \pm 6 \cdot 1 \\
79 \pm 7 \cdot 7\end{array}$ & $\begin{array}{l}92 \pm 5.9 \\
95 \pm 4.9\end{array}$ \\
\hline
\end{tabular}




\section{DISCUSSION}

The results described above for the interaction of cholesterol and cholestanol in oospore formation in Phytophthora cactorum have many points of resemblance with the interactions between auxins and anti-auxins in higher plants. The results are, essentially, that at high concentrations of cholesterol the addition of cholestanol decreases the number of oospores; at low concentrations of cholesterol the addition of some cholestanol increases the oospore number, but larger amounts of cholestanol decrease the number again. For the interaction of indolylacetic acid (IAA) and phenylbutyric acid (PBA) in oat coleoptile segments, Skoog, Schneider \& Malan (1942) found that at the lowest IAA concentration tested $(0.005 \mathrm{mg}$./1.) the addition of some PBA resulted in an increase in length greater than that obtained with IAA alone, but adding more PBA was inhibitory. At higher IAA concentrations ( $0.05 \mathrm{mg}$./1. and above) the growth with added PBA was always less than with IAA alone. Thimann \& Bonner (1948) found that high concentrations of tri-iodobenzoic acid inhibited, but low concentrations stimulated, growth induced by IAA. McRae et al. (1953) found that above a certain concentration of IAA the effect of adding 2,4-dichlorophenoxyacetic acid $(2,4-D)$ was inhibitory to elongation of coleoptile segments; below that concentration of IAA, adding 2,4-D was stimulatory.

By analogy with the model proposed by McRae et al. (I953), it may be supposed that there are a number of sites in the cellular structure of Phytophthora which can be occupied by cholesterol or cholestanol, and at which these sterols perform their function in controlling oospore development. Above a certain critical concentration of cholesterol the addition of cholestanol displaces cholesterol from these functional sites and the oospore number is decreased. Below the critical cholesterol concentration the addition of cholestanol first results in more sites being occupied. (For this to lead to more oospores being formed, cholestanol must have some real, though low, activity in promoting the reactions concerned.) Addition of more cholestanol, however, again results in the displacement of cholesterol from the functional sites and its replacement with the much less active cholestanol, with concomitant decrease in spore number.

The promotion of oospore development is not the only effect sterols have on Phytophthora; indeed the results of adding sterols to Phytophthora closely resemble those found on adding a vitamin to a fungus which has a partial deficiency for it. It would appear, however, that Phytophthora cannot synthesize any sterol at all (Elliott, Hendrie, Knights \& Parker, I964; Hendrix, 1966). In Phytophthora sterols are required in the medium for normal development of sporangia and zoospores (Hendrix, 1965; Chee \& Turner, 1965), and they also increase vegetative growth. The results reported in the present paper are consistent with the idea that there is only one kind of site occupiable by sterols and affecting oospore development, and different but related molecules can compete for these sites. However, it is possible that there is more than one kind of site which sterols can occupy. Cholestanol promotes vegetative growth only slightly less efficiently than cholesterol (Table 2; Elliott et al. 1966, Table 2), and both promote oogonium development, but they differ in their ability to promote the later oospore stages of the sexual process. The different specificities in molecular structure required for activity at these different stages suggest that sterols might exert their effect on them from different kinds of sites. The sterol requirement of insects can be met by a small amount of an essential sterol (cholesterol or 22-dehydrocholesterol) and a much 
larger amount of a sparing sterol (cholestanol and many other substances; Clark \& Bloch, 1959; Clayton \& Bloch, 1963). The essential and sparing sterols would appear to have different functions, and they occur in the tissues in states differing in the degree of esterification and in the ease with which they turn over with excess sterol in the diet (Lasser, Edwards \& Clayton, I966). This clearly suggests that in insects sterols occupy more than one kind of site, a similar situation to that suggested for Phytophthora.

If in fact there are at least two kinds of sites, the increase in oospore numbers effected by cholestanol at low cholesterol concentrations is more readily explained than if there were only one kind of site. It may also be that any one kind of site has a higher affinity for more active molecules than for less active ones, so that the sites controlling oospore development will be occupied by cholesterol rather than cholestanol, except when cholestanol is in great excess. Further, during growth of the fungus the oospore sites may be synthesized or become functional after those which control oogonium formation. Thus with cholesterol alone and at low concentration many oospore sites will be unoccupied, but with addition of cholestanol, cholesterol will be displaced from the sites concerned with vegetative growth and oogonium development, and the number of oospore sites occupied by cholesterol increased.

I am indebted to Dr B. A. Knights for analysis of the samples of cholesterol and cholestanol.

\section{REFERENCES}

Bowden, K., Heilborn, I. N., Jones, E. R. H. \& Weedon, B. C. L. (I946). Researches on acetylenic compounds. I. The preparation of acetylenic ketones by oxidation of acetylenic carbinols and glycols. J. chem. Soc. 1946, 39.

Chee, K. H. \& Turner, N. A. (1965). A steroid factor in pea (Pisum sativum L.) influencing growth and sporulation of Phytophthora cinnamoni Rnds. N.Z. Jl agric. Res. 8, 104.

ClaRk, A. J. \& BloCh, K. (1959). Function of sterols in Dermestes vulpinus. J. biol. Chem. 234, 2583.

Clayton, R. B. \& Bloch, K. (1963). Sterol utilization in the hide beetle, Dermestes vulpinus. J. biol. Chem. 238, 586.

Dixon, M. \& WeBb, E. C. (1964). Enzymes, 2nd edn. London: Longmans.

Elliott, C. G., Hendrie, M. R. \& Knights, B. A. (I966). The sterol requirement of Phytophthora cactorum. J.gen. Microbiol. 42, 425.

Elliott, C. G., Hendrie, M. R., Knights, B. A. \& Parker, W. (I964). A steroid growth factor requirement in a fungus. Nature, Lond. $203,427$.

Foster, R. J., MCRAE, D. H. \& BONNER, J. (1952). Auxin-induced growth inhibition, a natural consequence of two-point attachment. Proc. natn. Acad. Sci. U.S.A. 38, I0I4.

HENDRIX, J. W. (1965). Influence of sterols on growth and reproduction of Pythium and Phytophthora spp. Phytopathology 55, 790.

HendRIX, J. W. (1966). Inability of Pythium aphanidermatum and Phytophthora palmivora to incorporate acetate into digitonin-precipitable sterols. Mycologia 58, 307.

KNIGHTS, B. A. (1967). The analysis of plant sterols in faeces. Mem. Soc. Endocr. 16, 21 I.

LASSER, N. L., Edwards, A. M. \& Clayton, R. B. (I966). Distribution and dynamic state of sterols and steroids in the tissues of an insect, the roach Eurycotis floridana. J. Lipid Res. 7, 403.

MCRae, D. H., Foster, R. J. \& Bonner, J. (1953). Kinetics of auxin interaction. Pl. Physiol., Lancaster 28, 343.

Skoog, F., Schneider, C. L. \& Malan, P. (1942). Interaction of auxins in growth and inhibition. Am. J. Bot. 29, 568.

Thimann, K. V. \& Bonner, W. D. (1948). The action of tri-iodobenzoic acid on growth. Pl. Physiol., Lancaster 23, 158. 